\title{
Severe bullous systemic lupus erythematosus successfully treated with low dose rituximab: a case report from sub-Saharan Africa
}

\author{
A.A. Akpabio', B.I. Otike-Odibi ${ }^{2}$ \\ ${ }^{1}$ Internal Medicine Department, University of Uyo Teaching Hospital, Uyo, Nigeria; \\ 2Internal Medicine Department, University of Port Harcourt Teaching Hospital, Port-Harcourt,
}

Rivers State - Nigeria

\section{SUMMARY}

Bullous systemic lupus erythematosus (BSLE) is a blistering skin condition occurring in lupus patients. Although cutaneous manifestations are common in lupus (59-85\%), BSLE is rare, affecting $<5 \%$ of lupus patients with very few reported cases among Africans. Dapsone is effective in mild to moderate cases, but off-label standard-dose rituximab (1,000 mg 2 weeks apart) has led to good outcomes in refractory cases. We report the case of a 39-yearold African lady with BSLE, who was successfully treated with low-dose rituximab (500 mg 2 weeks apart), healing her skin lesions at one year post-treatment. Low-dose rituximab may be as effective as standard dosing for the treatment of severe bullous SLE and is recommended in low resource settings.

Key words: Bullous lupus; rituximab; Africans; case report.

Reumatismo, 2020; 72 (2): 115-119

\section{INTRODUCTION}

B ullous systemic lupus erythematosus (BSLE) is a blistering skin condition occurring in patients with lupus (1). Although cutaneous manifestations are common in lupus (59-85\%), BSLE is rare, affecting $<5 \%$ of lupus patients (2-4) with very few reported cases among Africans. From the reviewed literature on BSLE, cohorts from Europe, Latin America and Asia show a $100 \%$ female predominance with a median age at lupus diagnosis of 22.5-24 years (5-7).

Bullous SLE is typically a sub-epidermal, transient, tense, vesiculobullous eruption, which heals without scarring or milia. It affects mainly the trunk, upper extremities, neck, face, and vermillion border, but it can also affect the mucosa (2). Being an autoimmune neutrophilic blistering disease, it differs from cutaneous lupus in the absence of basal vacuolization (5). Antibodies to the non-collagenous ( $\mathrm{NC1}$ and 2) domain of type VII collagen and other antigens differentiate BSLE from other bullous condi- tions, such as bullous pemphigoid (BP), linear IgA bullous dermatosis, epidermolysis bullosa acquisita (EBA), and dermatitis herpetiformis (DH) (4).

Three classes of BSLE exist based on antibodies against collagen type VII or others (type I), yet undefined epitope(s) of the basement membrane (type II), or epidermis (type III) (8). It appears to be associated with systemic flares (mostly nephritis) (58 ), even though some have reported otherwise (3). Although dapsone is effective in addition to steroids and anti-malarial medications, off-label use of standard dose rituximab (1,000 mg 2 weeks apart) has led to successful outcomes in refractory cases (9). We present the case of a 39-year-old African lady with BSLE successfully treated with low-dose rituximab (500 mg 2 weeks apart).

\section{CASE REPORT}

Mrs CFB, a 39-year-old lady was first diagnosed with SLE by a physician in Novem-
Corresponding author: Akpabio Akanimo Akpabio Internal Medicine Department University of Uyo Teaching Hospital, Uyo, Nigeria

E-mail: petersakpabio@yahoo.com 
ber 2017 after a month of recurrent fever, fatigue, hair loss, polyarthritis, frothy urine, facial and leg swelling. She also had generalized bullous rashes, oral ulcers, photosensitivity and two ulcers on the left leg occurring at herbal incision sites. Investigations showed ANA 1:5120 (speckled), dsDNA $300 \mathrm{IU} / \mathrm{mL}(>18)$ and mild pericardial effusion on echocardiography. She was started on oral prednisolone, hydroxychloroquine (HCQ) and sunscreen with improvement in symptoms. Mycophenolate mofetil (MMF) $1.5 \mathrm{~g}$ twice daily was initiated by the nephrologists.

After 4 months on treatment, she improved, $1+$ proteinuria disappeared and she stopped taking her medications thinking she was cured. However, symptoms reappeared a month later (May 2018). She was worried about the unsightly bullous rashes over her face, trunk and arms as well as the leg ulcers. Rashes were unresponsive to steroids (oral and topical), dapsone and mycophenolate mofetil, necessitating referral from her dermatologist to a rheumatologist for a more potent treatment.

Physical examination showed that this nonsmoking obese lady (BMI $29.3 \mathrm{~kg} / \mathrm{m}^{2}$ ) had mild pallor, facial puffiness and bilateral leg edema. No fever, oral ulcers, synovitis or asterixis were noted. She had a pulse rate of $72 \mathrm{~b} / \mathrm{min}$, blood pressure of $120 / 80$ $\mathrm{mmHg}$, respiratory rate of $20 \mathrm{c} / \mathrm{min}$ with normal cardiovascular and chest findings. Multiple small bullae $(1 \mathrm{~cm}$ diameter, in various stages of healing) were seen over the arms, legs, chest and back with facial sparing. Two ulcers were also noted - a larger $8 \times 6 \mathrm{~cm}$ oval ulcer on the medial third of her leg and a smaller $1 \mathrm{~cm} \mathrm{x} 1 \mathrm{~cm}$ round ulcer on the dorsum of her foot both with unhealthy granulation tissue and slough.

A biopsy of one of the bullous lesions performed by the referring dermatologist had shown a predominantly ulcerated epidermis, focally preserved with clusters of neutrophils and lymphocytes within the horny layer accompanied by acanthosis in other areas. There were dense lymphocytic infiltrates in a lichenoid (band-like) pattern with accompanying basal vacuolization within the superficial dermis and nodular clusters within the dermis. The overall picture was compatible with SLE.

An impression of SLE flare with bullous lupus, nephritis and infected vasculitic ulcers was made. Investigations showed: hematocrit $35 \%$, leucocytes $9800 \mathrm{~mm}^{3}$, platelets $295,000 \mathrm{~mm}^{3}, \mathrm{ESR}=48 \mathrm{~mm} / \mathrm{hr}$, creatinine $53 \mu \mathrm{mol} / \mathrm{l}$, urea $4.2 \mathrm{mmol} / \mathrm{l}$, sodium $137 \mathrm{mmol} / \mathrm{l}$, potassium $3.7 \mathrm{mmol} / 1$, chloride $102 \mathrm{mmol} / \mathrm{l}$ and bicarbonate 27 $\mathrm{mmol} / \mathrm{l}$. She had a normal urinalysis with 6-10 white cells/hpf on microscopy, but no red blood cells, casts or crystals. Renal ultrasound was normal. Biopsy showed Class I nephritis with mild patchy active chronic interstitial nephritis, arteriolar hyalinosis and essentially normal glomeruli on light and immunofluorescence microscopy. Amyloid deposits were absent and staining for IgM, IgG and complement were all negative.

The urine protein-creatinine ratio (UPCR) was $124 \mathrm{mg} /$ day $(<15)$ with an estimated GFR (CKD-EPI) of $115 \mathrm{mls} /$ minute ( $>90)$. A wound swab showed Staphylococcus aureus sensitive to clindamycin, meropenem and imipenem. She restarted the treatment with MMF tablets $500 \mathrm{mg}$ to be taken twice daily (increased to $1 \mathrm{~g}$ twice daily after 2 weeks), oral prednisolone $20 \mathrm{mg}$ daily, HCQ tablets $200 \mathrm{mg}$ daily, dapsone tablets $50 \mathrm{mg}$ daily, lisinopril tablets $5 \mathrm{mg}$ daily, betamethasone cream twice daily, sunscreen SPF 50 as well as daily wound dressing and twice daily oral clindamycin $600 \mathrm{mg}$ and she was counseled on the need for biologics.

The bullae persisted, despite the abovementioned therapies for 3 months with at least 10 new bullae appearing and rupturing daily (Figure 1). She finally purchased and received IV rituximab (Mabthera ${ }^{\circledR}$ Roche) in February 2019 at a dose of 500 mg 2 weeks apart (due to cost considerations). Skin lesions resolved completely after 3 months with residual hyperpigmentation and remained stable at 1 year posttreatment (Figure 2).

She recently underwent venous stripping of varicose veins on account of the poorly healing left leg ulcers and is currently back 


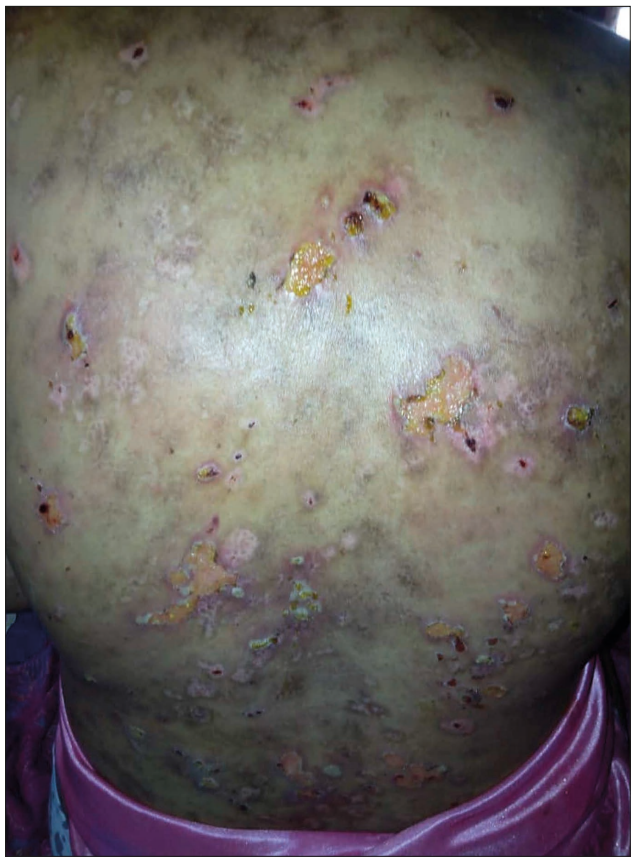

Figure 1 - Pre-treatment bullous systemic lupus erythematosus.

on MMF tablets $1 \mathrm{~g}$ twice daily and other medications with normal urinalysis, electrolytes (Creatinine $88 \mu \mathrm{mol} / \mathrm{l}$ ), normal complement $[\mathrm{C} 3=101.1 \mathrm{mg} / \mathrm{dL}$ (90-180), $\mathrm{C} 4=26 \mathrm{mg} / \mathrm{dL}$ (10-40)], HBA1 ${ }_{\mathrm{C}} 4.8 \%$, $\mathrm{PCV} 34 \%, \mathrm{ESR}=45 \mathrm{~mm} / \mathrm{hr}$ and elevated UPCR $344 \mathrm{mg} /$ day noted after taking some herbal treatments. So far dipstick protein is negative and UPCR remained within a range of 124-344 mg/day throughout her treatment. The patient is being monitored with regular follow-up visits to her rheumatologist and dermatologist.

\section{DISCUSSION}

Bullous SLE is an acquired blistering disease in lupus patients reportedly occurring in African American females usually between the second and the fourth decade $(2,9)$ like in our patient, although few cases have been reported in children (10). Relatively very few cases of BSLE have been identified among large lupus cohorts in Asia $(0.19 \%)$ and Latin America $(0.41 \%)$ (6). Among black Africans, reports are rare with only one case identified

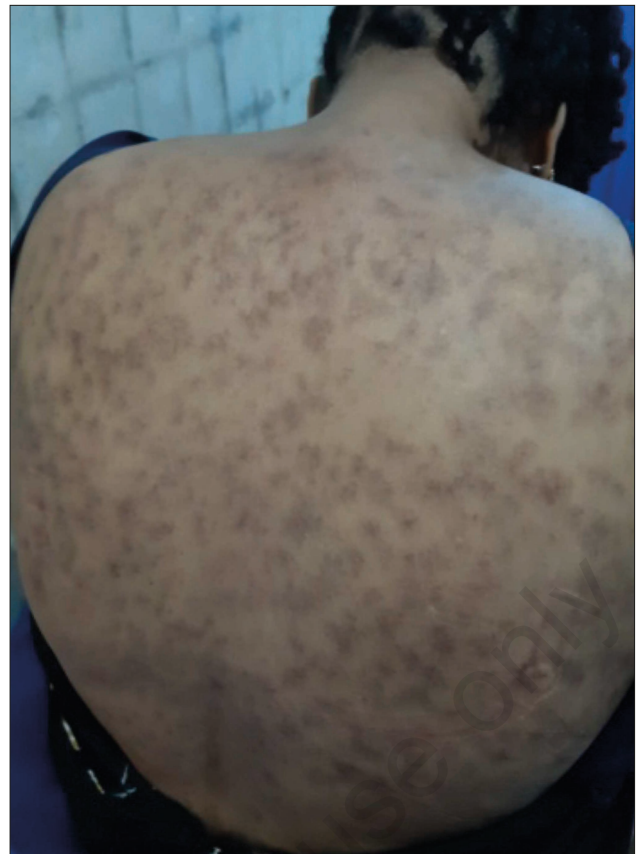

Figure 2 - Post-treatment bullous systemic lupus erythematosus.

among 47 Nigerian cutaneous lupus patients by Ayanlowo et al. (11).

Diagnostic criteria for BSLE were first described by Camisa and Sharma in 1983 (12) and included:

1) diagnosis of SLE based on ACR criteria;

2) vesicles and/or bullae;

3) histopathologic features similar to dermatitis herpetiformis;

4) direct immunofluorescence with $\operatorname{IgG}$ and/or IgM and often IgA at the basement membrane zone (BMZ);

5) indirect immunofluorescence testing that can be negative or positive for circulating autoantibodies against the BMZ.

Subsequent criteria (8) subdivide BSLE into three classes based on the presence of antibodies to type VII collagen and location of the antibodies against the BMZ. Although our case met only some of the clinical criteria, a detailed evaluation with skin immunofluorescence was not feasible in our resource-limited setting.

It is important to note that bullous SLE lesions can be differentiated from other cutaneous forms of SLE due to the absence of 
basal vacuolization (5). Although in our case the skin biopsy reportedly showed basal vacuolization, this may have occurred because biopsy was taken from an erosion on the right upper hand with part of the normal skin - possibly an erosion from subacute SLE. Basal vacuolization typically results from interface dermatitis, which is characteristic of cutaneous lupus.

The pathogenesis of BSLE involves autoantibodies directed against non-collagenous domain type 1 and 2 (NC1 and NC2) of type VII collagen, found in the basement membrane zone (BMZ). Recently, antibodies against laminin-5, laminin- 6 and bullous pemphigoid antigen 1 have been investigated (1). Although similar antigens exist in other bullous conditions, such as epidermolysis bullosa acquisita (EBA) and dermatitis herpetiformis (DH), bullous SLE stands out due to its occurrence in the context of SLE.

The onset of bullous lesions may sometimes correlate with systemic disease, such as nephritis $(2,13)$, like in our patient, although this is not always the case (3). Nephritis has been observed in 50-90\% $(5,7)$ of BSLE patients reflecting a more severe phenotype, which can be difficult to treat $(13,14)$. Additionally, BSLE has occurred with hematologic features (7), neuropsychiatric symptoms (5) and even pregnancy (15).

A striking response to dapsone is usually seen in BSLE both in adults and children $(3,10)$. Other immunosuppressive medications, such as azathioprine, methotrexate and mycophenolate mofetil, may also be used with variable efficacy especially in patients with systemic flares or intolerance to dapsone (3-5). Cyclophosphamide was avoided in our patient for fertility reasons. In severe cases like ours there may be no response to dapsone and/or other immunosuppressants necessitating treatment escalation to biologics. This lack of response to dapsone as well as the occurrence of mild anemia were the reasons for the lower dose of $50 \mathrm{mg}$ daily used compared to the standard maintenance dose of $50 \mathrm{mg}$ twice daily $(1-1.5 \mathrm{mg} / \mathrm{kg} /$ day $)$ previously given by the dermatologist.

Regarding the dose of MMF, apart from the initial induction dose of $1.5 \mathrm{~g}$ twice daily given by the nephrologists, which she took for 4 months with the disappearance of proteinuria, a low dose of $500 \mathrm{mg}$ twice daily for 2 weeks was given when the treatment was restarted due to the presence of a wound infection. Following its resolution, MMF was quickly increased to the standard maintenance dose of $1 \mathrm{~g}$ twice daily, although this did not result in an improvement in the skin lesions or vasculitic ulcers. Off-label use of rituximab, a monoclonal antibody against CD-20, has been reported to be effective in refractory BSLE cases, where it is thought to deplete antibody producing B cells. A standard dose of $1000 \mathrm{mg}$ 2 weeks apart has been shown to offer long-term efficacy in patients with refractory $\operatorname{BSLE}(2,3,9)$.

In our sub-Saharan African setting, patients pay out-of-pocket due to inadequate health insurance, thus making biologic therapy difficult. The cost of rituximab is quite prohibitive (about $\$ 1000$ per $500 \mathrm{mg}$ vial at the time of our patient's treatment), therefore our patient could only afford two 500 mg treatments two weeks apart (a total of $1000 \mathrm{mg}$ ). Following delete this rituximab therapy, there was complete resolution of skin lesions persisting at 1 year post-treatment. In conclusion, bullous SLE is a rare acquired blistering skin condition in lupus patients and, although standard therapy with dapsone and immunosuppressants may be effective, low-dose rituximab is beneficial in refractory cases and for resource-poor settings.

\section{Acknowledgements}

The authors wish to acknowledge Dr Emediong S. Asuka for his assistance with rituximab administration.

\section{REEERENCES}

1. Chan LS, Lapiere J-C, Chen M, et al. Bullous systemic lupus erythematosus with autoantibodies recognizing multiple skin basement membrane components, bullous pemphigoid Antigen 1, Laminin-5, Laminin-6, and Type VII Collagen. Archiv Dermatol. 1999; 135: 569-73.

2. Contestable JJ, Edhegard KD, Meyerle JH. Bullous systemic lupus erythematosus: a re- 
view and update to diagnosis and treatment. Am J Clin Dermatol. 2014; 15: 517-24.

3. Duan L, Chen L, Zhong S, et al. Treatment of bullous systemic lupus erythematosus. J Immunol Res. 2015: 167064.

4. Rutnin S, Chanprapaph K. Vesiculobullous diseases in relation to lupus erythematosus. Clin Cosmet Investig Dermatol. 2019; 12: 653-67.

5. de Risi-Pugliese T, Cohen Aubart F, Haroche $\mathrm{J}$, et al. Clinical, histological, immunological presentations and outcomes of bullous systemic lupus erythematosus: $10 \mathrm{New}$ cases and a literature review of 118 cases. Semin Arthritis Rheum. 2018; 48: 83-9.

6. Pons-Estel GJ, Quintana R, Alarcon GS, et al. A 12-year retrospective review of bullous systemic lupus erythematosus in cutaneous and systemic lupus erythematosus patients. Lupus 2018; 27: 1753-4.

7. Chanprapaph K, Sawatwarakul S, Vachiramon V. A 12-year retrospective review of bullous systemic lupus erythematosus in cutaneous and systemic lupus erythematosus patients. Lupus 2017; 26: 1278-84.

8. Yell JA, Allen J, Wojnarowska F, et al. Bullous systemic lupus erythematosus: revised criteria for diagnosis. Br J Dermatol. 1995; 132: 921-8.
9. Anyanwu CO, Ang CC, Werth VP. Oral mucosal involvement in bullous lupus. Arthritis Rheum. 2013; 65: 2622.

10. Lenka U, Sengupta D, Sardar S, Ghosh S. Bullous systemic lupus erythematosus: An uncommon diagnosis in an 8-year-old girl. Indian J Paediatr Dermatol. 2016; 17: 139-41.

11. Ayanlowo O, Ima-Edomwonyi U, Adelowo O, et al. Cutaneous manifestations in systemic lupus erythematosus patients attending a tertiary hospital in Nigeria. Afr J Rheumatol. 2018; 6: 44-9.

12. Camisa C, Sharma HM. Vesiculobullous systemic lupus erythematosus. Report of two cases and a review of the literature. J Am Acad Dermatol. 1983; 9: 924-33.

13. Momen T, Madihi Y. Bullous systemic lupus erythematosus and lupus nephritis in a young girl. Oman Med J 2016; 31: 453-5.

14. Ng YY, Chang IT, Chen TW, et al. Concomitant lupus nephritis and bullous eruption in systemic lupus erythematosus. Nephrol Dial Transplant 1999; 14: 1739-43.

15. Vinicki JP, Juarez MA, Muzzio I, et al. Bullous systemic lupus erythematosus in a pregnant woman associated with lupus nephritis responding to dapsone. J Rheumatol Arthritic Dis. 2016; 1: 1-3. 